\title{
A General Search for New Phenomena at HERA
}

\author{
Gerhard Brandt ${ }^{1}$ \\ 1- Deutsches Elektronen-Synchrotron \\ Notkestr. 85, 22761 Hamburg - Germany
}

\begin{abstract}
A model-independent search for deviations from the Standard Model prediction using the full $e^{ \pm} p$ data sample collected by the H1 experiment at HERA is presented. All event topologies involving isolated electrons, photons, muons, neutrinos and jets with transverse momenta $P_{T}$ above $20 \mathrm{GeV}$ are investigated in a single analysis. Events are assigned to exclusive classes according to their final state. A dedicated algorithm is used to search for deviations from the Standard Model in the distributions of the scalar sum of transverse momenta or the invariant mass of final state particles and to quantify their significance. Variables related to angular distributions and energy sharing between final state particles are also introduced to study the final state topologies. No significant deviation from the Standard Model expectation is observed in the phase space covered by this analysis.
\end{abstract}

\section{Analysis Method}

At HERA electron-proton collisions ${ }^{\mathrm{a}}$ at centre-of-mass energies of up to $319 \mathrm{GeV}$ provide a testing ground for the Standard Model (SM) complementary to $e^{+} e^{-}$and $p \bar{p}$ scattering. In this talk [1] a purely signature based search for new physics beyond the Standard Model in the HERA data, corresponding to an integrated luminosity of $463 \mathrm{pb}^{-1}$, is presented [2].

The analysis investigates all possible high- $P_{T}$ topologies with at least two particles: electrons $(\mathrm{e})$, photons $(\gamma)$, muons $(\mu)$ and jets $(\mathrm{j})$, identified in a common phase space $10^{\circ}<\theta<140^{\circ}$ and $P_{T}>20 \mathrm{GeV}$. A neutrino $(\nu)$ is identified if the event has missing transverse momentum above $20 \mathrm{GeV}$ and $\left(E-P_{z}\right)<48 \mathrm{GeV}$. All particles are required to be isolated with respect to each other by a minimum distance $R>1$ in $(\eta, \phi)$ space, where $\eta$ is the pseudorapidity and $\phi$ is the azimuthal angle. The events are sorted depending on the number and types of particles into exclusive event classes. The event yields for all event classes are presented for the data and SM expectation in figure 1 for $e^{+} p$ and $e^{-} p$ collisions. All event classes with observed data events or with a SM expectation greater than 0.01 events are shown. Events are observed in 27 classes and a good description of the number of observed data events by the SM prediction is seen in each class.

The distributions of the scalar sum of transverse momenta $\sum P_{T}$ and of the invariant mass $M_{\text {all }}$ of all bodies are investigated. The data are in agreement with the SM prediction. In particular, multiple jets topologies, which are sensitive to QCD radiation, are well described by the simulation. The final state topologies are also evaluated in terms of angular distributions and energy ratios, which are sensitive to spin and decay properties of hypothetical high mass particles. Variables used to study the decomposition of the final states, inspired by topological analyses of multi-jet events [4], are defined. In each event a leading particle is selected based on particle type and transverse momentum. The variable $\cos \theta_{\text {lead }}^{*}$ is defined as the cosine of the polar angle of the leading particle relative to the incident

\footnotetext{
"In the following the term "electron" is used generically to refer to both electrons and positrons, unless otherwise stated.
} 


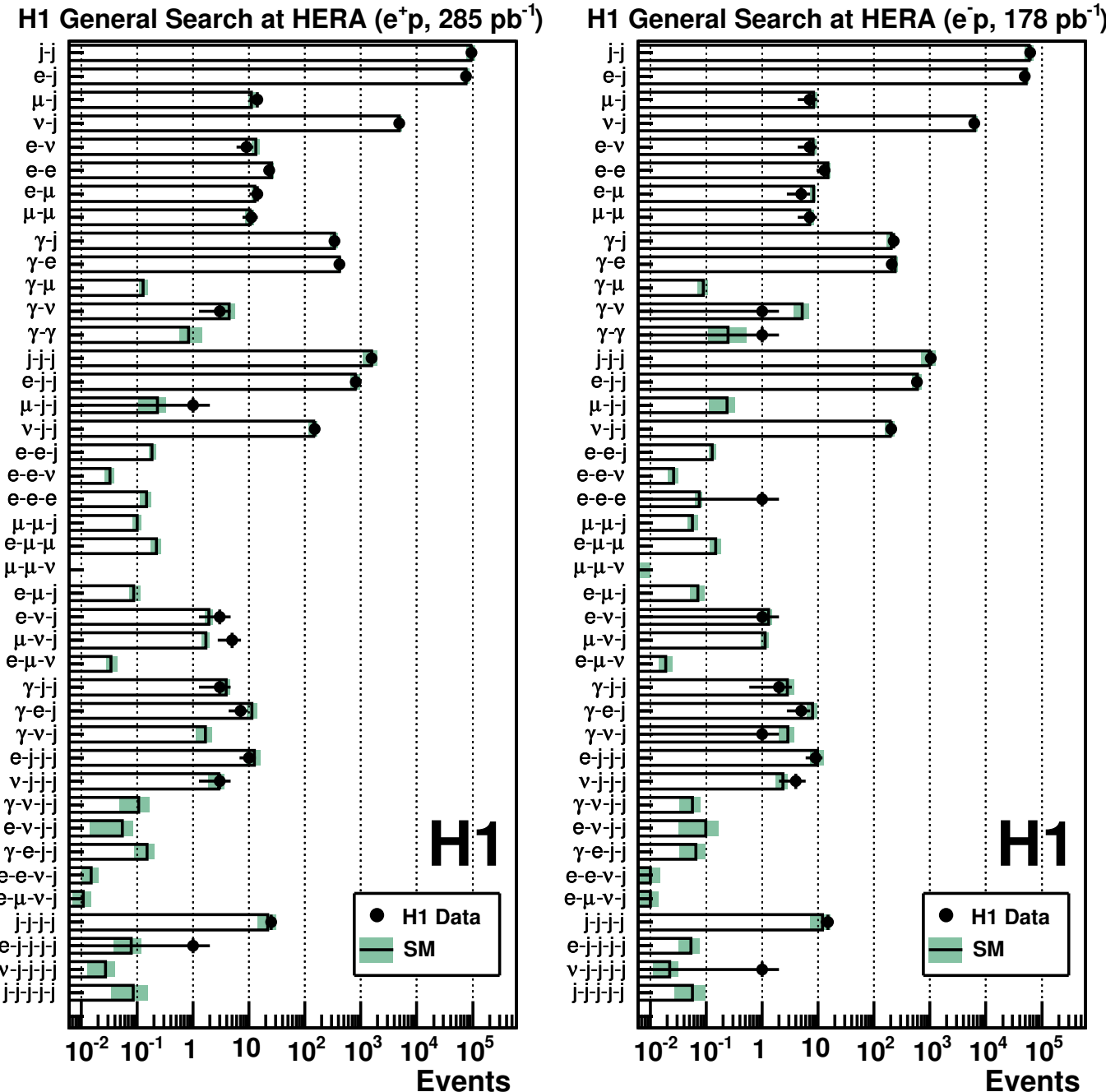

Figure 1: The data and the SM expectation for all event classes with observed data events or a SM expectation greater than 0.01 events for $e^{+} p$ collisions (left) and $e^{-} p$ collisions (right). The error bands on the predictions include model uncertainties and experimental systematic errors added in quadrature. 
proton in the centre-of-mass frame defined by all particles. For events with two bodies, the $\cos \theta_{\text {lead }}^{*}$ distribution is related to the underlying $2 \rightarrow 2$ matrix element. Therefore, the angular distribution of a particle coming from the decay of a new resonance may be markedly different from that of particles produced in SM processes (see for example [5]). The variable $X_{\text {lead }}$ is the energy fraction of the leading particle and is defined for systems with three or more particles as

$$
X_{\text {lead }}=\frac{2 E_{\text {lead }}^{*}}{\sum_{i} E_{i}^{*}}
$$

where the sum runs over all particles energies, and $E_{\text {lead }}^{*}$ and $E_{i}^{*}$ are calculated in the centreof-mass frame of all particles. For final states with more than two particles, $X_{\text {lead }}$ is related to the dynamics of a possible multi-body decay of a new particle. The sensitivity of these two variables $\cos \theta_{\text {lead }}^{*}$ and $X_{\text {lead }}$ to new physics is tested using different MC samples of exotic processes. It has been verified that SM and exotic events exhibit different spectra in these two variables, two examples of which are given in figure 2. The event topology is well described by the SM.
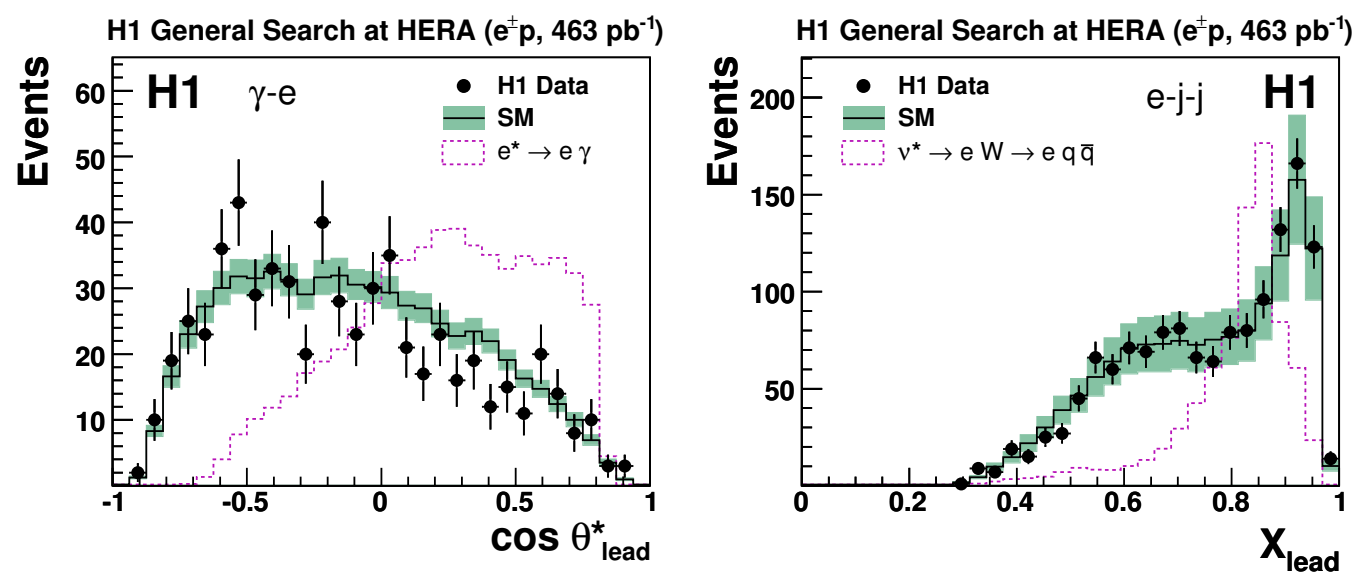

Figure 2: The $\cos \theta_{\text {lead }}^{*}$ distribution in the $\gamma$ - $e$ event class (a) and the $X_{\text {lead }}$ distribution in the ejj event class (b). The points correspond to the observed data events and the histograms to the SM expectation. The error bands on the SM prediction include model uncertainties and experimental systematic errors added in quadrature. The dashed line represents, with an arbitrary normalisation, the distribution corresponding to an exotic resonance with a mass of $200 \mathrm{GeV}\left(e^{*}[6]\right.$ (left) and $\nu^{*}[7]$ (right)).

\section{Statistical Interpretation}

In order to quantify the level of agreement between the data and the SM expectation and to identify regions of possible deviations, a dedicated search algorithm developed for a general search in HERA I data at the $\mathrm{H} 1$ experiment is employed [3]. Regions in the $\sum P_{T}, M_{\text {all }}, \cos \theta_{\text {lead }}^{*}$ and $X_{\text {lead }}$ distributions are defined as a sets of connected histogram 
bins. The number of data events $\left(N_{o b s}\right)$, the SM expectation $\left(N_{S M}\right)$ and its total systematic uncertainty $\left(\delta N_{S M}\right)$ are calculated for each region. A statistical estimator $p$ to judge which region is of most interest is defined via

$$
p=\left\{\begin{array}{lll}
A \int_{0}^{\infty} d b G\left(b ; N_{S M}, \delta N_{S M}\right) & \sum_{i=N_{o b s}}^{\infty} \frac{e^{-b} b^{i}}{i !} & \text { if } N_{o b s} \geq N_{S M} \\
A \int_{0}^{\infty} d b G\left(b ; N_{S M}, \delta N_{S M}\right) & \sum_{i=0}^{N_{o b s}} \frac{e^{-b} b^{i}}{i !} & \text { if } N_{o b s}<N_{S M}
\end{array}\right.
$$

based on the usual Poisson probability for small backgrounds $b$ in bins $i$ convoluted with a Gaussian pdf $G$ to account for non negligible systematic uncertainties, and $A$ is a factor to ensure normalisation to unity. The region of greatest deviation is the region having the smallest $p$-value, $p_{\min }$. A probability $\hat{P}$ that a fluctuation with a $p$-value at least as small as $p_{\min }$ occurs anywhere in the distribution is estimated using Monte Carlo experiments based on the SM expectation. Finally, the overall degree of agreement with the SM is further quantified by doing many Monte Carlo (MC) experiments simulating a full set of hypothetical data histograms. The probability of observing an event class with a given $\hat{P}$ value is calculated as above and the distribution of $\hat{P}^{S M}$ values obtained from all MC experiments is formed. This distribution is compared to the $\hat{P}$ values observed in the data. The most significant deviation from SM predictions is measured in $e^{+} p$ collisions in the $e$-e event class with $\hat{P}=0.0035$. In the corresponding region $\left(110<M_{\text {all }}<120 \mathrm{GeV}\right)$ five data events are found while $0.43 \pm 0.04$ are expected. The global probability to find in the $e^{+} p$ data at least one class with a $\hat{P}$ value smaller than observed in the $e$-e event class is $12 \%$.

\section{Summary}

A model-independent search for deviations from the Standard Model prediction using the full $e^{ \pm} p$ data sample collected by the $\mathrm{H} 1$ experiment at HERA has been performed. No significant deviation from the SM expectation has been found, demonstrating the good understanding of high- $P_{T}$ processes at HERA. This search constitutes the most comprehensive general search for new physics at high-energy colliders.

\section{References}

[1] Slides: http://indico. cern. ch/contributionDisplay . py? contribId=106\&sessionId=2\&conf Id=53294

[2] F. D. Aaron et al. [H1 Collaboration], "A General Search for New Phenomena at HERA", Phys. Lett. B 674 (2009) 257 [arXiv:0901.0507 [hep-ex]].

[3] A. Aktas et al. [H1 Collaboration], "A general search for new phenomena in ep scattering at HERA", Phys. Lett. B 602 (2004) 14 [hep-ex/0408044].

[4] S. Geer and T. Asakawa, "The Analysis of Multijet Events Produced at High Energy Hadron Colliders", Phys. Rev. D 53 (1996) 4793 [hep-ph/9510351].

[5] A. Aktas et al. [H1 Collaboration], Phys. Lett. B 629 (2005) 9 [hep-ex/0506044].

[6] F. D. Aaron et al. [H1 Collaboration], Phys. Lett. B 666 (2008) 131 [arXiv:0805.4530 [hep-ex]].

[7] F. D. Aaron et al. [H1 Collaboration], Phys. Lett. B 663 (2008) 382 [arXiv:0802.1858 [hep-ex]]. 